\title{
Research on Compressible Flow around Cylinder and Truncated Cone with LES Method
}

\author{
Meng Zhao ${ }^{1, \text { a }}$, Jun Mao ${ }^{1, b}$ and Guowei Yang ${ }^{2, c}$ \\ ${ }^{1}$ School of Civil Engineering, Beijing Jiaotong University, China 100044 \\ ${ }^{2}$ Institute of Mechanics, Chinese Academy of Sciences, China 100190 \\ a09115287@bjtu.edu.cn, bjmao@bjtu.edu.cn, ’gwyang@imech.ac.cn
}

Keywords: Flow around circular cylinder, LES, Vortex Street, Flow filed

\begin{abstract}
Numerical simulation with the large eddy simulation, filter function, and Smagorinsky sub-grid scale model is adopted to simulate the compressible flow around a bluff body finite length circular cylinder and truncated cone in high Reynolds number. The law of the drag coefficient, lift coefficient and pressure coefficient obtained from models with various cross-wind speeds was discussed. Process of the vortex generated, shed and dissipated was analyzed and the relationship between the press filed, velocity filed and vortex filed was also analyzed. Average value of the drag coefficient, lift coefficient and pressure coefficient of the circle cylinder in subcritical region are greater than truncated cone. Values of pressure coefficient on the windward side of all the models are consistent. However, it various widely on the leeward side, even on the end face of the cone and cylinder.
\end{abstract}

\section{Introduction}

Cylinder is used widely in the industry and daily life. Flow around a body is a common physical phenomenon such as air flowing around air plane, pantograph, pier and cable. Flowing around a variable cross section cylinder is also very common. Compressible turbulence is a research hotspot by the development of high speed train, aviation and scramjet. Drag reduction and flow control technical in these filed are related firmly to the solutions of the flow with high Reynolds number, unsteady and compressible turbulence. Vortex shed periodically behind the circular cylinder in some conditions which was named as Karman Vortex Street ${ }^{[1]-[6]}$. The periodically shedding of the vortex would force on the circular cylinder in the vertical direction of the flow, which may induce vibration on the circular cylinder and lead to fatigue failure.

\section{Numerical simulation method of compressible turbulence}

Turbulence is a complex 3D unsteady flow with rotation, which includes various dimensions of vortex with random motion. Methods of numerical simulation include DNS, RANS, LES and DES. Large eddy simulation resolves large scales of the flow field solution, which allowing better fidelity than alternative approaches such as RANS methods. It also models the smallest scales of the solution, which makes the computational cost for practical engineering systems with complex geometry or flow configurations attainable using supercomputers. LES method was adopted in this research ${ }^{[7]-[9]}$.

By filtering the vortex smaller than a certain scale from the partial differential equations, only vortex greater than that scale was involved, the smaller vortex was solved from the additional equation. After this process, the $u_{\mathrm{i}}$ in N-Sin equation was as follow:

$\overline{u_{i}}(x, t)=\frac{1}{\Delta^{3}} \iint_{D} G\left(x-x^{\prime}\right) u\left(x^{\prime}, t\right) d x^{\prime}$

Where $\Delta$ is the filter scale. By adopt the Box-filter as follow: 


$$
\begin{aligned}
& G(x)=1 \cdots \cdots \cdots \cdots \cdot\left|x_{i}\right| \leq \Delta / 2 \\
& G(x)=0 \cdots \cdots \cdots \cdots \cdot\left|x_{i}\right|>\Delta / 2
\end{aligned}
$$

The N-S equation becomes was as follow:

$\frac{\partial \bar{u}_{i}}{\partial t}+\frac{\partial\left(\bar{u}_{i} \bar{u}_{j}\right)}{\partial x_{j}}=-\frac{1}{\rho} \frac{\partial \bar{p}}{\partial x}+v \frac{\partial^{2} \bar{u}_{j}}{\partial x_{i} \partial x_{j}}+\frac{\partial \bar{\tau}_{i j}}{\partial x_{j}}$

$\bar{\tau}_{i j}=-\left(\overline{u_{i} u_{j}}-\bar{u}_{i} \bar{u}_{j}\right)$ is the subgrid Reynolds stress, which means the influence of the small scale vortex to the large scale vortex.

The Smagorinsky-Lilly subgrid model was adopted which as follow:

$$
\bar{\tau}_{i j}=\left(C_{s} \Delta\right)^{2} \bar{S}_{i j}\left(2 \bar{S}_{i j} \bar{S}_{j j}\right)^{\frac{1}{2}}-\frac{1}{3} \bar{\tau}_{k k} \delta_{i j}
$$

Where the $S_{i j}$ is the rate-of-strain tensor and $C_{s}$ is a constant.

\section{Establishment of physical model and mathematical model}

The finite length circular cylinder and truncated cone are established. The change of cylindrical cross - sectional areas is conveniently specified by the apex angle. The geometric models of finite length circular cylinder and truncated cone are shown in Fig.1.
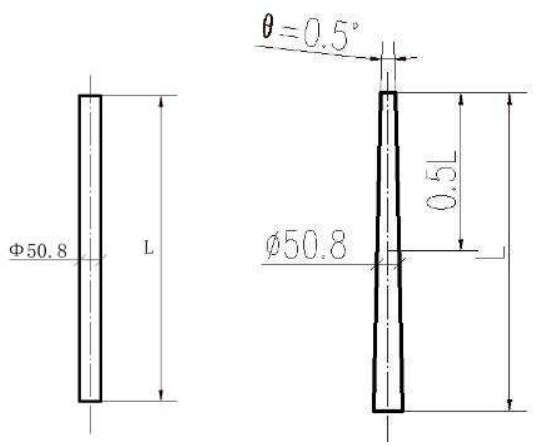

Fig.1 The finite length circular cylinder and truncated cone

The domain size upstream the cylinder is 10 times of cylinder diameter and the domain size downstream is more than 50 times of the cylinder diameter, which is large enough to eliminate the influence of boundary. Grids around the cylinder and in the wake are refined. The grid of the domain is shown in Fig.2.
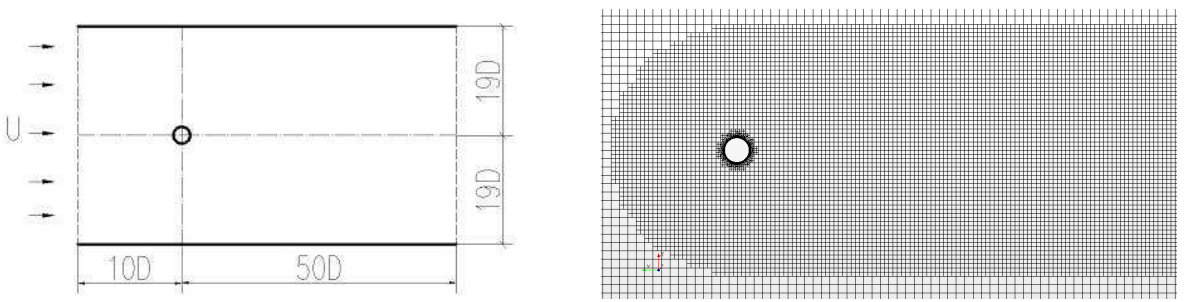

Fig. 2 The computational domain and grid of the model

The setting of boundary conditions has an important influence on the numerical simulation result. Cross-wind was added according to the common engineering condition, the setting of boundary conditions is as follow: the entrance velocity along the $X$ direction is $97.22 \mathrm{~m} / \mathrm{s}$; the entrance velocity along the $\mathrm{Y}$ direction is $15 \mathrm{~m} / \mathrm{s}$; the outlet pressure is $0 \mathrm{~Pa}$; the boundary of cylinder is no-slip and others are slip. The Reynolds number is between 2.2E5 and 3.5E5. 


\section{Analysis of simulation result}

The law of the drag coefficient, lift coefficient and pressure coefficient obtained from models with various cross-wind speeds was discussed. Process of the vortex generated, shed and dissipated was analyzed and the relationship between the press filed, velocity filed and vortex filed was also analyzed.

Analysis of the drag coefficient distribution of circular cylinder and truncated cone in time-domain and spectrum are shown in Fig.3 and Fig.4. The result shows that the average value and amplitude value of drag coefficient of the truncated cone in subcritical region are greater than circle cylinder. The laws of periodic change of two models are nearly the same, and the low frequency characteristic of truncated cone is obvious.

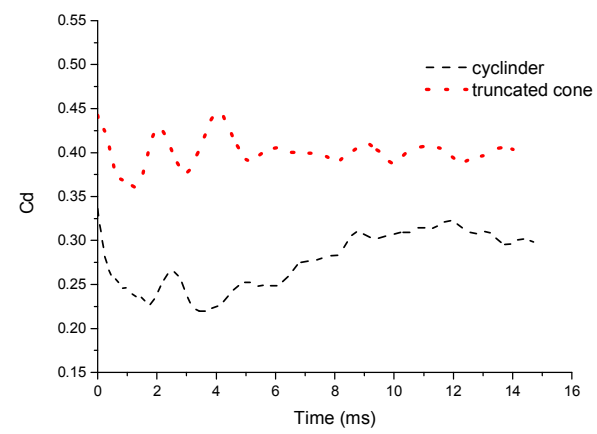

Fig.3 The drag coefficient in time-domain

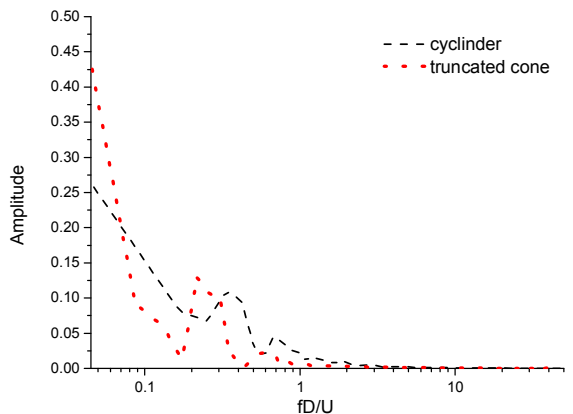

Fig.4 The drag coefficient in Spectrum

Analysis of the drag coefficient distribution of circular cylinder and truncated cone in time-domain and spectrum are shown in Fig.5 and Fig.6. The average value and amplitude value of lift coefficient of the truncated cone in subcritical region are greater than circle cylinder.

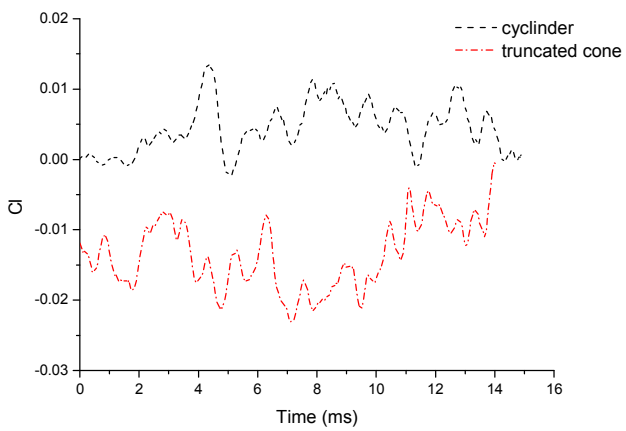

Fig.5 The lift coefficient in time-domain

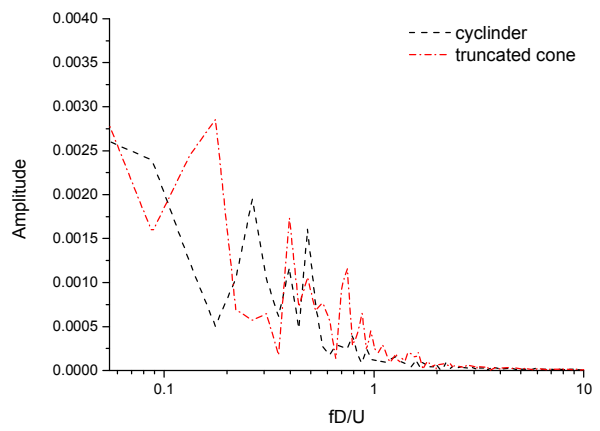

Fig.6 The lift coefficient in Spectrum

Six cross-sections named from section I to section VII were established along the lengthwise direction to investigate the pressure distribution along the circle, which as shown in Fig.7.

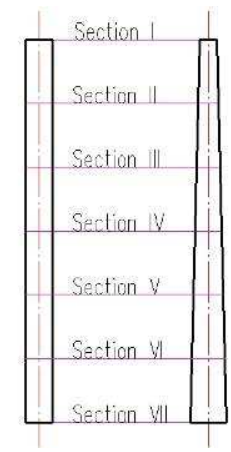

Fig.7 Cross-sections of the model 
Circle pressure coefficient of all circle cylinders and truncated cones were shown in Fig. 8 and Fig.9. Circle pressure coefficients of the two models along the lengthwise direction are nearly the same. An inflection point appeared at the $90^{\circ}$ position. Circle pressure coefficients of the two models on the end section were shown in Fig.10 and Fig.11, which were influenced by the end section seriously. As [9] shown, separation point was in the inflection point position of the pressure coefficient. Apex angle is not a key factor to the pressure coefficient.

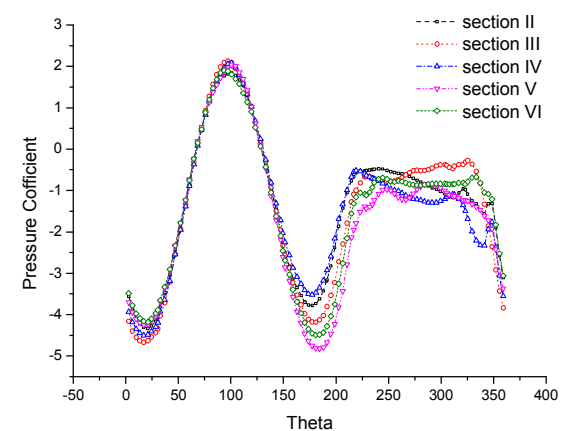

Fig. 8 Time history of the drag coefficient

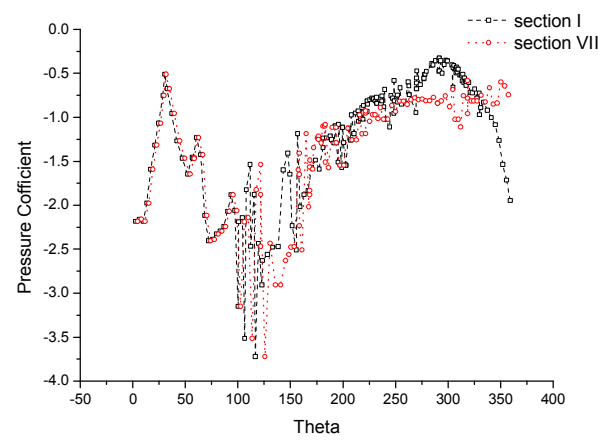

Fig.10 Time history of the drag coefficient

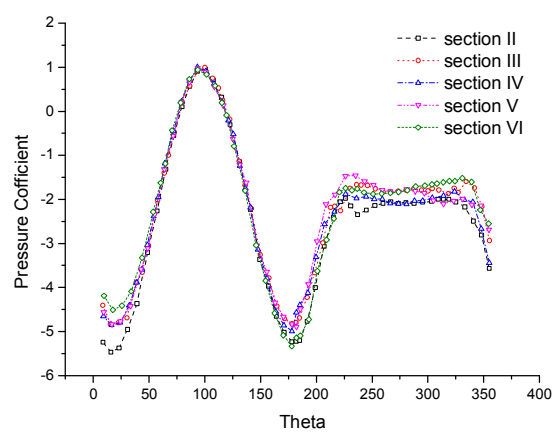

Fig.9 Spectrum analysis of the drag coefficient

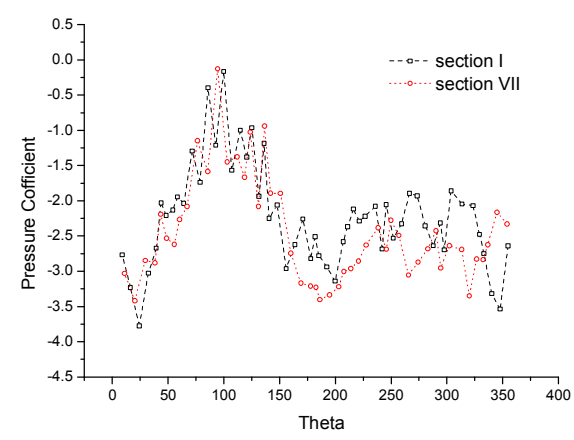

Fig.11 Spectrum analysis of the drag coefficient

\section{The distribution of flow field}

The velocity distribution of circular cylinder and truncated cone is shown in Fig.12 and Fig.13. As shown in the figure, a stagnation point appeared in the first time step and two high speed regions appeared on both side of the circle cylinder. High speed regions on both side of the circle cylinder expanded rapidly as the low speed region behind the circle cylinder diffused downstream. A large low speed region occurred finally behind the circle cylinder. Compared to the circle cylinder, the low speed region behind the truncated cone was larger.

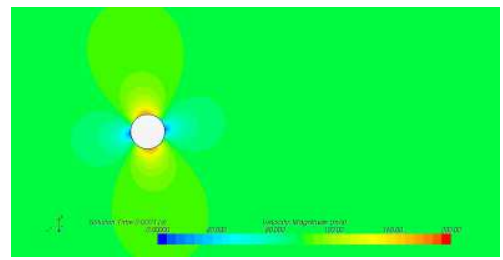

(a) $\mathrm{t}=0.1 \mathrm{~ms}$

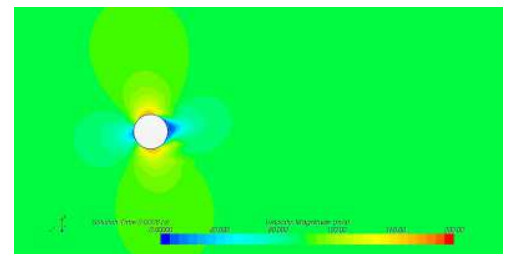

(b) $\mathrm{t}=1 \mathrm{~ms}$

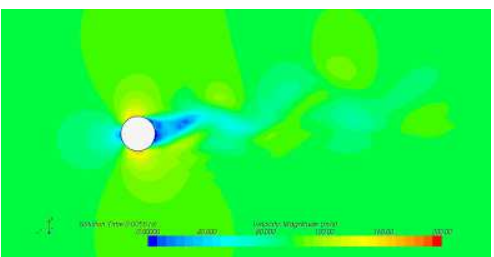

(c) $\mathrm{t}=5 \mathrm{~ms}$

Fig. 12 The velocity distribution of circular cylinder 


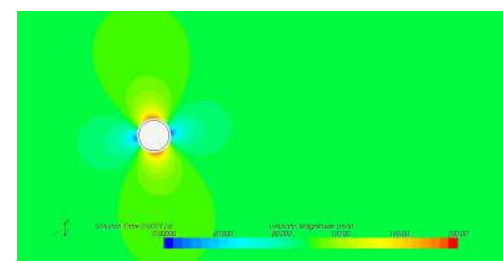

(a) $\mathrm{t}=0.1 \mathrm{~ms}$

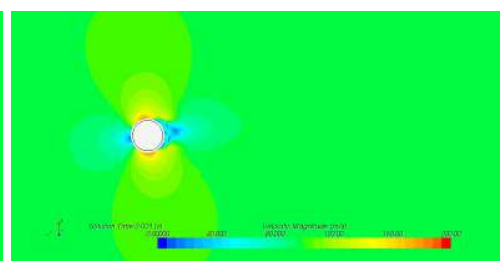

(b) $\mathrm{t}=1 \mathrm{~ms}$

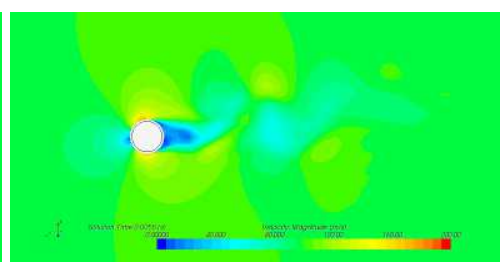

(c) $\mathrm{t}=5 \mathrm{~ms}$

Fig. 13 The velocity distribution of truncated cone

Process of the vortex generated, shed and dissipated of circular cylinder and truncated cone is shown in Fig.14 and Fig.15, the relationship between the press filed, velocity filed and vortex filed is also shown in the figures.

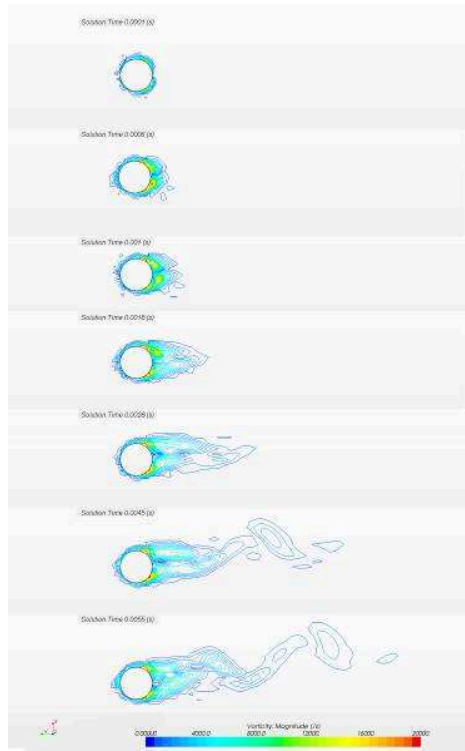

Fig.14 Vortex of the circular cylinder

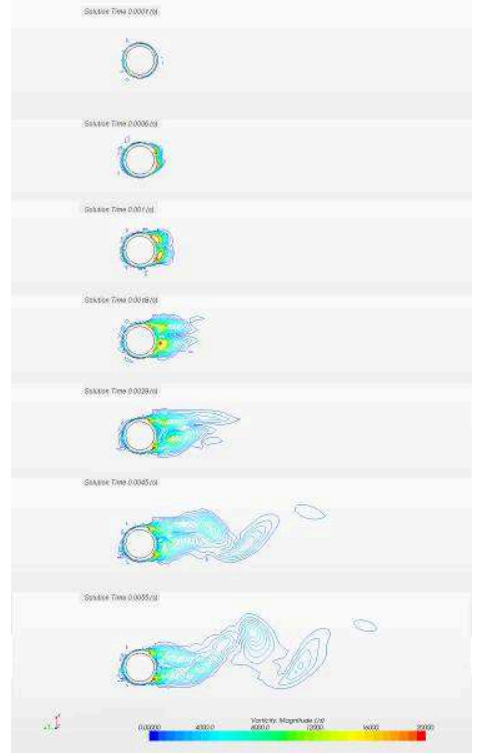

Fig.15 Vortex of the truncated cone

\section{Conclusions}

This paper calculated the performance parameters of compressible fluid by different geometric models, compared the different results due to circular cylinder and truncated cone, and fully discussed the influence of the calculation models in analyzing the flow with high Reynolds number. The average value amplitude value of the drag coefficient and lift coefficient of the truncated cone. in subcritical region are greater than circle cylinder; of all the models, the distribution of pressure coefficient showed significant periodicity, inflection points were around the $90^{\circ}$ position. Values of pressure coefficient on the windward side of all the models are consistent. However, it various widely on the leeward side, even on the end face of the cone and cylinder. A low pressure region occurred because of the blockage of the circle cylinder or the truncated cone, which was the center of the vortex. The vortex flow backwards and a larger low pressure region formatted finally.

\section{Acknowledgements}

This work was financially supported by the National Natural Science Foundation of China (51278032), National Key Technology R\&D Program of the Ministry of Science and Technology (GTYZ200902). 


\section{References}

[1] Jiwei YANG, Xiaoli FU. Research on the flow around a cylinder [J]. China Water Transport, 2008,8(5):156-158.(in Chinese)

[2] ZDRAVKOVICH M M. Review-review of flow interference between two circular cylinders in various arrangements [J]. Journal of Fluid Engineering, 1977, 12: 618-33.

[3] Xiaohe JIA. Large Eddy Simulation of Flow around One and Two Circular Cylinders [D].Beijing: Shanghai Jiaotong University, 2008: 1-5. (in Chinese)

[4] Shouying LI, Ming GU. Numerical Simulation for Flow around Perpendicular[J], Acta Aerodynamica Sinica, 2006,23(2): 222-227 .(in Chinese)

[5] Jun LIAO, Sirui JING. Numerical Simulation of Flow around Two Cylinder at high Reynolds number[J]. Jouranal of Hydrodynamics， 2001,16(1): 101-109.(in Chinese)

[6] Xiongliang YAO, Yuanyuan FANG, Shaoshi DAI et al. Three-dimensional Numerical Simulation of the F1ow past a Circular Cylinder Based on LES Method [J]. Jouranal of Hydrodynamics, 2007,22(5): 564-571. (in Chinese)

[7] Wenquan TAO. Numerical Heat Transfer[M]. Second Edition. Xi'an. Xi'an Jiao Tong University Press.2001:1-18.(in Chinese)

[8] Wen JIN, YANG Zhigang. Turbulence Structure and theLarge Eddy Simulation [J]. Energy and environment, 2005, 4: 11-14. (in Chinese)

[9] NISHIMUA H, TANIKE Y. Aerodynamic characteristics of fluctuating forces on a circular cylinder [J]. Journal of wind Engineering and Industrial Aerodynamics, 2001, 89:713-723 
Advanced Construction Technologies

10.4028/www.scientific.net/AMR.919-921

Research on Compressible Flow around Cylinder and Truncated Cone with LES Method 10.4028/www.scientific.net/AMR.919-921.210 